\title{
The ESO Nearby Abell Cluster Survey
}

\section{The orbits of the different types of galaxies in rich clusters ${ }^{\star}, \star \star$}

\author{
A. Biviano ${ }^{1}$ and P. Katgert ${ }^{2}$ \\ 1 INAF - Osservatorio Astronomico di Trieste, Italy \\ e-mail: biviano@ts.astro.it \\ 2 Sterrewacht Leiden, The Netherlands
}

Received 17 May 2004 / Accepted 3 June 2004

\begin{abstract}
We study the orbits of the various types of galaxies observed in the ESO Nearby Abell Cluster Survey. We combine the observed kinematics and projected distributions of galaxies of various types with an estimate of the mass density profile of the ensemble cluster to derive velocity-anisotropy profiles. Galaxies within and outside substructures are considered separately. Among the galaxies outside substructures we distinguish four classes, on the basis of their projected phase-space distributions. These classes are: the brightest ellipticals (with $M_{R} \leq-22+5 \log h$ ), the other ellipticals together with the S0's, the earlytype spirals $(\mathrm{Sa}-\mathrm{Sb})$, and the late-type spirals and irregulars (Sbc-Irr) together with the emission-line galaxies (except those of early morphology). The mass profile was determined from the distribution and kinematics of the early-type (i.e. elliptical and S0) galaxies outside substructures; the latter were assumed to be on isotropic orbits, which is supported by the shape of their velocity distribution. The projected distribution and kinematics of the galaxies of other types are used to search for equilibrium solutions in the gravitational potential derived from the early-type galaxies. We apply the method described by Binney \& Mamon as implemented by Solanes \& Salvador-Solé to derive, to our knowledge for the first time, the velocity anisotropy profiles of all galaxy classes individually (except, of course, the early-type class). We check the validity of the solutions for $\beta^{\prime}(r) \equiv\left[\left\langle v_{\mathrm{r}}^{2}\right\rangle(r) /\left\langle v_{\mathrm{t}}^{2}\right\rangle(r)\right]^{1 / 2}$, where $\left\langle v_{\mathrm{r}}^{2}\right\rangle(r)$ and $\left\langle v_{\mathrm{t}}^{2}\right\rangle(r)$ are the mean squared components of the radial and tangential velocity, respectively, by comparing the observed and predicted velocity-dispersion profiles. For the brightest ellipticals we are not able to construct equilibrium solutions. This is most likely the result of the formation history and the special location of these galaxies at the centres of their clusters. For both the early and the late spirals, as well as for the galaxies in substructures, the data allow equilibrium solutions. The data for the early spirals are consistent with isotropic orbits $\left(\beta^{\prime}(r) \equiv 1\right)$, although there is an apparent radial anisotropy at $\simeq 0.45 r_{200}$. For the late spirals an equilibrium solution with isotropic orbits is rejected by the data at the $>99 \%$ confidence level. While $\beta^{\prime}(r) \approx 1$ within $0.7 r_{200}, \beta^{\prime}$ increases linearly with radius to a value $\simeq 1.8$ at $1.5 r_{200}$. Taken at face value, the data for the galaxies in substructures indicate that isotropic solutions are not acceptable, and tangential orbits are indicated. Even though the details of the tangential anisotropy remain to be determined, the general conclusion appears robust. We briefly discuss the possible implications of these velocity-anisotropy profiles for current ideas of the evolution and transformation of galaxies in clusters.
\end{abstract}

Key words. galaxies: clusters: general - galaxies: kinematics and dynamics - cosmology: observations

\section{Introduction}

The orbital characteristics of the various types of galaxies in present-day clusters can give unique information about the evolution of the clusters themselves, and about the formation and evolution of their member galaxies. This is because clusters are still accreting galaxies from their surroundings, and the details of this accretion process provide constraints for theories of cluster evolution. In addition, the orbits of the various types of galaxies yield clues about the history of their accretion onto the cluster, and about the evolutionary relationships between them.

* Based on observations collected at the European Southern Observatory (La Silla, Chile).

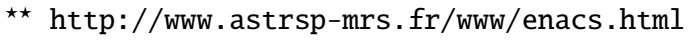

The idea of shells of collapsing material around clusters has been around since the work of Gunn \& Gott (1972). This work stimulated several investigations, and indirect evidence for the infall of spirals into clusters has been accumulating over the years. Moss \& Dickens (1977) were the first to observe a difference in the velocity dispersions of cluster earlytype and late-type galaxies, followed by Sodré et al. (1989), and Biviano et al. (1992). The different projected phase-space distributions of early-type (red) and late-type (blue) galaxies was clearly established by Colless \& Dunn (1996) and Biviano et al. (1996) in the Coma cluster, while Carlberg et al. (1997a) found it in the CNOC clusters at $z \approx 0.3$. The effect was studied in detail for the clusters observed in the ESO Nearby Abell Cluster Survey (ENACS, hereafter; de Theije \& Katgert 1999, Paper VI; Biviano et al. 2002, Paper XI), as well as in other 
clusters (see, e.g., Adami et al. 1998a). Galaxies with emission lines (ELG) provide a rather extreme example of the effect. The ELG are less centrally concentrated and have a higher dispersion of line-of-sight velocity than the galaxies without emission lines. This was first shown by Mohr et al. (1996) for the A576 cluster, and clearly demonstrated by Biviano et al. (1997, Paper III) for the ENACS clusters.

These results suggest mildly radial orbits of the late-type galaxies with emission lines, probably in combination with first approach to the central dense core. This interpretation would be consistent with the presence of the line-emitting gas which is unlikely to "survive" when the galaxy crosses the cluster core. Indeed, Pryor \& Geller (1984) tried to constrain the orbits of HI-deficient galaxies by noting that cluster-core crossing is a necessary condition for gas stripping, and Solanes et al. (2001) noted that the velocity-dispersion profile of HI-deficient galaxies is quite steep, suggestive of radial orbits. Support for the scenario of spiral infall into clusters comes from the the analyses of the Tully-Fisher distance-velocity diagram (Tully \& Shaya 1984; Gavazzi et al. 1991). Indirect support comes from the numerical simulations that show that dark matter particles have a moderate radial velocity anisotropy, which increases out to the virial radius (e.g. Tormen et al. 1997; Ghigna et al. 1998; Diaferio 1999). Radio or X-ray trails of cluster galaxies can also be used to constrain their orbits (Merrifield 1998).

In the absence of full dynamical modelling, the analysis of the galaxy spatial distribution and kinematics can only suggest, but not really constrain, the nature of cluster galaxy orbits. This is because the projected spatial distribution, kinematics and mass model are coupled. So far, only a few full dynamical analyses of the orbital distribution of cluster galaxies exist. One reason for this is the relative paucity of detailed data on the kinematics and distributions of cluster galaxies, in particular if several galaxy classes are considered. Another reason is that the orbital characteristics can only be inferred from the observed kinematics and distributions if the mass density profile of the cluster is known. The latter must be derived either from the distribution of light (with assumptions about the radial variation of the mass-to-light ratio), or from the projected phase-space distribution of that subset of the galaxies for which the properties of their full phase-space distribution can be estimated independently.

A first dynamical analysis of the orbits of cluster galaxies was made for the Coma cluster by Kent \& Gunn (1982). Using several analytical mass models, these authors concluded that the galaxy orbits in the Coma cluster cannot be primarily radial, so that even at large radii a significant part of the kinetic energy of the galaxies must be in the tangential direction. They noted that the range of the predicted velocity dispersions of the galaxies of different morphological types was only half that which is observed. Although a marginal result, this could indicate different distribution functions for the galaxies of different types, and not just different energy distributions. Merritt (1987) used the same data to estimate the orbital anisotropy of the galaxies in the Coma cluster, for various assumptions about the radial dependence of the mass-to-light ratio.

More recent dynamical modelling of galaxy clusters has led to the conclusion that the orbits of early-type galaxies are quasi-isotropic, while those of late-type galaxies are moderately radial (e.g. Natarajan \& Kneib 1996; Carlberg et al. 1997b; Mahdavi et al. 1999; Biviano 2002; Łokas \& Mamon 2003). This picture is not supported by the analysis of Ramírez \& de Souza (1998) who studied the deviations from Gaussianity of the overall distribution of the line-of-sight velocities of the galaxies. These authors concluded that the orbits of ellipticals are close to radial, while spirals would have more isotropic orbits. However, van der Marel et al. (2000) and Biviano (2002) argue that the conclusion of Ramírez \& de Souza is most likely due to erroneous assumptions in their modelling.

One of the most extensive dynamical analyses so far was done for 14 "regular" galaxy clusters from the CNOC (Carlberg et al. 1997b,c). Adopting ad hoc functional forms for the $3 \mathrm{D}$ number density, the mean squared components of the radial velocity, and the velocity anisotropy profile, Carlberg et al. $(1997 b, 1997 \mathrm{c})$ concluded that the velocity anisotropy is zero or at most mildly radial. The CNOC data were re-analysed by van der Marel et al. (2000), who used the method developed by van der Marel (1994), assuming a three-parameter family of mass-density profiles, and a set of constant values for the velocity anisotropy, to determine the parameters in the mass-profile model from the best fit to the line-of-sight velocity dispersion profile. More recently, from the analysis of the projected phasespace distribution of $\sim 15000$ galaxies in the infall regions of eight nearby clusters (the CAIRNS project), Rines et al. (2003) concluded that galaxy orbits are consistent with being isotropic within the virial radius. Note that neither van der Marel et al. (2000), nor Rines et al. (2003) distinguished among different cluster galaxy populations.

In this paper we study the galaxy orbits in an ensemble cluster of 3056 galaxy members of 59 clusters observed in the ENACS. We use the "inversion" of the Jeans equation of stellar dynamics, as derived by Binney \& Mamon (1982), and we apply the solution method given by Solanes \& Salvador-Solé (1990, hereafter $\mathrm{S}^{2}$ ). The analysis requires the mass profile $M(<r)$, for which we use the estimate derived by Katgert et al. (2004, Paper XII) for the same ensemble cluster. Preliminary results were discussed by Biviano et al. (1999, 2003, 2004), Mazure et al. (2000), Biviano (2002), and Biviano \& Katgert (2003).

In Sect. 2 we summarize the data that we use, describe the different classes of cluster galaxies, and the construction of the "ensemble cluster". In Sect. 3 we discuss the observational basis for our analysis, i.e. the projected and de-projected number density profiles, and the velocity-dispersion profiles of the various galaxy classes. In Sect. 4 we summarize the observed mass profile and the model fits that we used in the analysis of the orbits. In Sect. 5 we summarize the inversion procedure by which we derived the velocity-anisotropy profiles for the brightest ellipticals, the early spirals, the late spirals, and the galaxies in substructures. In Sect. 6 we describe the results of the analysis, and in Sect. 7 we discuss the implications of the results for ideas about the evolution of the clusters themselves, and about the formation and evolution of galaxies in clusters. Our summary and conclusions are given in Sect. 8. Throughout this paper we use $H_{0}=100 \mathrm{hm} \mathrm{s}^{-1} \mathrm{Mpc}^{-1}$. 


\section{The data, the galaxy classes, and the ensemble cluster}

Our analysis of the orbits of galaxies in rich clusters is based on data obtained in the context of the ENACS. Katgert et al. (1996, 1998, Papers I and V of this series, respectively) describe the multi-object fiber spectroscopy with the 3.6-m telescope at La Silla, as well as the photometry of the 5634 galaxies in 107 rich, nearby $(z \lesssim 0.1)$ Abell clusters. After the spectroscopic survey was done, a long-term programme of CCD-imaging with the Dutch 92-cm telescope at La Silla was carried out which has yielded photometrically calibrated images for 2295 ENACS galaxies. Thomas (2004, Paper VIII) has used those images to derive morphological types, with which he also refined and recalibrated the galaxy classification based on the ENACS spectra, as carried out previously in Paper VI.

The morphological types derived by Thomas were supplemented with morphological types from the literature, and those were combined with the recalibrated spectral types from Paper VI into a single classification scheme. This has yielded galaxy types for 4884 ENACS galaxies, of which $56 \%$ are purely morphological, $35 \%$ are purely spectroscopic, and $6 \%$ are a combination of both. The remaining 3\% had an early morphological type (E or S0) but showed emission lines in the spectrum. With these galaxy types, Thomas \& Katgert (2004, Paper X) studied the morphology-radius and morphologydensity relations. These galaxy types also form the basis of the study of morphology and luminosity segregation (Paper XI).

In Paper XI the galaxy classes were defined that must be distinguished because they have different phase-space distributions. In particular, this applies to galaxies within and outside substructures. The membership of a given galaxy to a substructure was determined using a slightly modified version of the test of Dressler \& Shectman (1988). In this test, a quantity $\delta$ was computed for each galaxy, designed to indicate when the neighbourhood of the galaxy is characterized by a different average velocity, and/or a smaller velocity dispersion than the cluster mean values (see Paper XI for details). Galaxies with $\delta \leq 1.8$ were shown to have a very small probability of belonging to substructures. On the other hand, only two thirds of the galaxies with $\delta>1.8$ really belong to substructures. In the present paper, we use $\delta=1.8$ to separate galaxies within substructures from galaxies outside substructures. However, we also checked our results for the galaxies in substructures with $\delta>2.2$. Clearly, the $\delta>2.2$ sample is smaller than the $\delta>1.8$ sample, but there is less contamination by galaxies outside substructures. The results for the $\delta>1.8$ sample are confirmed from the $\delta>2.2$ sample. Therefore, for the sake of simplicity, in the rest of this paper we only refer to "galaxies in substructures" (or, more simply, "Subs", in the following), meaning galaxies with $\delta>1.8$, keeping in mind that the same results apply for the galaxies with $\delta>2.2$.

In Paper XI we showed that four classes of cluster galaxies must be distinguished among the galaxies outside substructures, on the basis of their projected phase-space distributions. These are: (i) the brightest ellipticals (with $M_{R} \leq-22+5 \log h$ ), which we will refer to as " $E_{\mathrm{br}}$ ", (ii) the other ellipticals together with the S0 galaxies (to be referred to as "Early"), (iii) the early spirals (Sa-Sb), which we will denote by " $S_{\mathrm{e}}$ ", and (iv) the late spirals and irregulars (Sbc-Irr) together with the ELG (except those with early morphology), or " $S_{1}$ " for short.

Summarizing, we consider 5 classes of cluster galaxies: $E_{\mathrm{br}}$, Early, $S_{\mathrm{e}}, S_{1}$, and Subs, containing 34, 1129, 177, 328, and 686 galaxies, respectively. As explained in Appendix B.1 of Paper XII, corrections for incomplete azimuthal coverage in the spectroscopic observations and sampling incompleteness had to be applied in the construction of the number density profiles. In order to keep these correction factors sufficiently small, galaxies located in poorly-sampled regions were not used and those have not been included in the numbers given above.

The present analysis requires that data for several clusters are combined into an ensemble cluster, to yield sufficient statistical weight. If clusters form a homologous set, the ensemble cluster effectively represents each of the clusters, provided that the correct scaling was applied. Support for the assumption of homology comes from the existence of a fundamental plane that relates some of the cluster global properties (Schaeffer et al. 1993; Adami et al. 1998b, Paper IV; Lanzoni et al. 2004). As shown by Beisbart et al. (2001), clusters with substructure deviate from that fundamental plane. Instead of eliminating all clusters with signs of substructure, we have chosen to consider separately those galaxies that are in substructures.

As in Papers XI and XII, we combined the data for 59 clusters with $z<0.1$, each with at least 20 member galaxies with ENACS redshifts, and with galaxy types for at least $80 \%$ of the members (see Table A.1 in Paper XI). The resulting ensemble cluster contains 3056 member galaxies, for 2948 (or 96\%) of which a galaxy type is available. The selection of cluster members was based on the method of den Hartog \& Katgert (1996), and its application to the ensemble cluster is summarized in Appendix A of Paper XII. We refer to Papers XI and XII for details on the way in which the data for many clusters were combined. Those details concern the uniform method for the determination of cluster centres, and the correct scaling of projected distances from the cluster centres, $R$ (with $r_{200}$ ), and of relative line-of-sight velocities (with the global line-of-sight velocity dispersion $\sigma_{p}$ ). The scaling with $r_{200}$ ensures that we avoid, as much as possible, mixing inner virialized cluster regions with external non-virialized cluster regions. Note that the scaling factors $r_{200}$ and $\sigma_{p}$ are computed using all cluster members.

We assume that the ensemble cluster is spherically symmetric, not rotating, and in a steady state. As discussed at length in Appendix C of Paper XII, these are reasonable assumptions for our ensemble cluster.

\section{The number-density and velocity-dispersion profiles}

The observational basis for our study of the orbits of galaxies in clusters is provided by the projected number-density profiles $I(R)$, and the velocity-dispersion profiles $\sigma_{p}(R)$ for the 5 galaxy classes that we consider, viz. " $E_{\mathrm{br}}$ ", "Early", " $S_{\mathrm{e}}$ ", " $S_{1}$ ", and "Subs" (see Sect. 2). Here we summarize the steps involved in the determination of these profiles, and their de-projection. Full details can be found in Appendix B of Paper XII. 


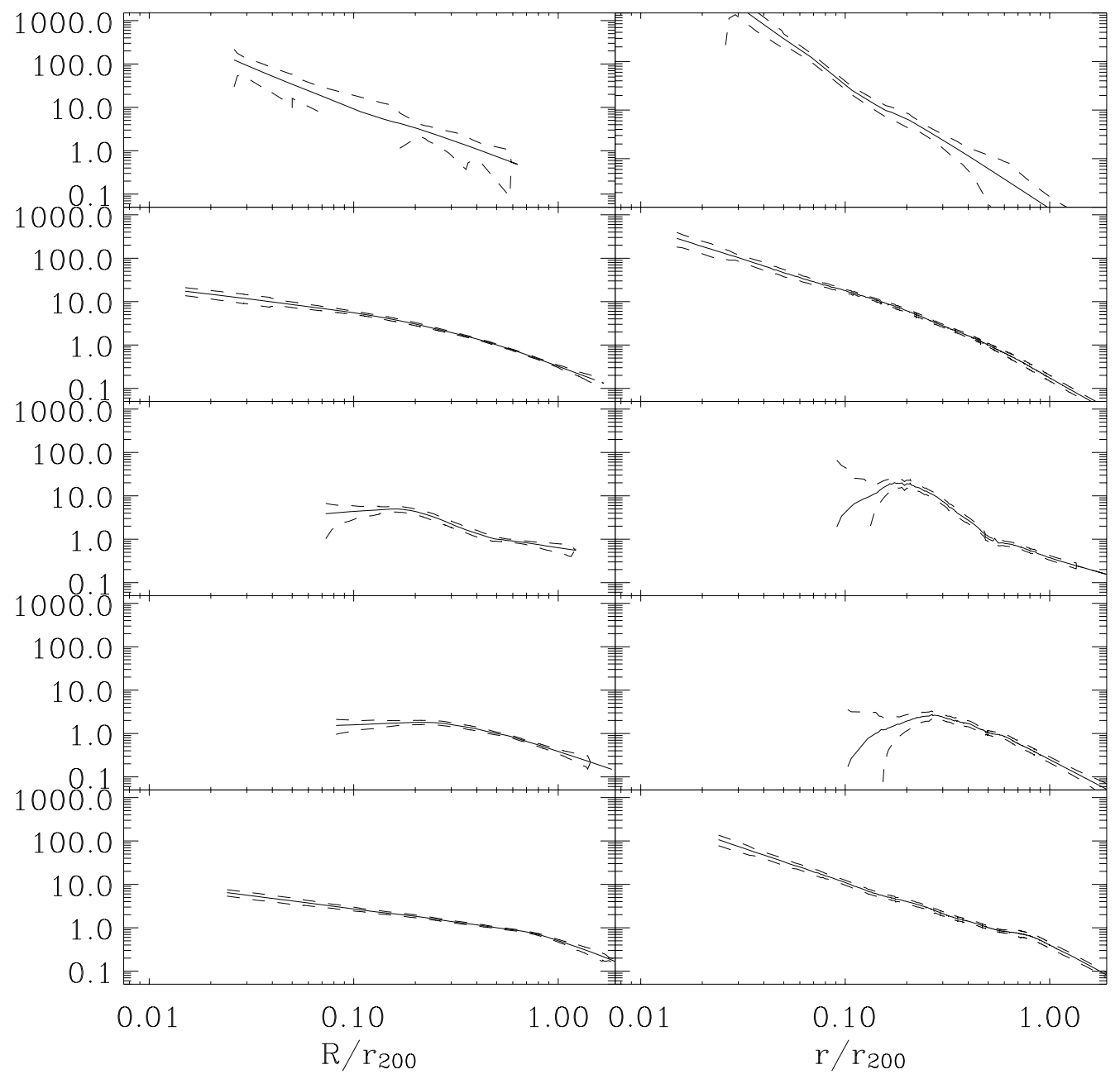

Fig. 1. Left: the best LOWESS estimate (solid line) of the projected number density $I(R)$, within the 1- $\sigma$ confidence interval determined from bootstrap resamplings (dotted lines), for each of the 5 galaxy classes, from top to bottom: $E_{\mathrm{br}}$, Early, $S_{\mathrm{e}}, S_{1}$, Subs. The scale on the $y$-axis is arbitrary. Right: same as left panel, but for the de-projected number density $v(r)$.

For the application of the Jeans equation - to derive the mass profile -, and its "inversion" - to derive the velocityanisotropy profile -, smooth estimates of number density profiles, velocity-dispersion profiles and combinations thereof are required. We used the LOWESS technique (e.g. Gebhardt et al. $1994)$ to obtain smooth estimates of $I(R)$ and $\sigma_{p}(R)$. Whereas Gebhardt et al. (1994) applied the LOWESS technique only to the estimation of a velocity dispersion profile, we also developed a variant that produces a smooth estimate of the number density profile.

The LOWESS technique yields estimates of $I(R)$ and $\sigma_{p}(R)$ at the projected distance $R$ of each galaxy. These estimates are based on a weighted linear fit to local estimates of projected density and velocity dispersion. The linear fits typically involve between 30 and $80 \%$ of the data points, but with a weight that drops steeply away from the galaxy in question. The number density profiles, $I(R)$ 's, were corrected for sampling incompleteness, assuming axial symmetry. Bootstrap resamplings yield estimates of the $68 \%$ confidence limits (approximately $1 \sigma$-errors) of the LOWESS estimate. The projected number density profiles $I(R)$ of the 5 galaxy classes are shown in the left-hand panels of Fig. 1, together with their $68 \%$ confidence limits.

In the Jeans equation as well as in its "inversion" one also needs the de-projected 3D number density $v(r)$. In the righthand panels of Fig. 1 we show the $v(r)$-profiles, as derived by de-projection via the Abel integral:

$v(r)=-\frac{1}{\pi} \int_{r}^{\infty} \frac{\mathrm{d} I}{\mathrm{~d} R} \frac{\mathrm{d} R}{\sqrt{R^{2}-r^{2}}}$

This de-projection involves no assumptions other than spherical symmetry, the extrapolation of $I(R)$ beyond the last measured point towards large radii (for which we assume a tidal radius of $6.67 r_{200}$ ), and continuity of $I(R)$ and its derivative at the last measured point. We checked that the de-projected profiles are essentially independent of the detailed form of the extrapolated $I(R)$.

In Fig. 2 we show the projected velocity dispersion profiles of the 5 galaxy classes as determined with the LOWESS technique. In the same figure, we also show binned estimates of the velocity dispersion, where the value of $\sigma_{p}(R)$ in each radial bin is computed using the robust biweight estimator (see Beers et al. 1990). 


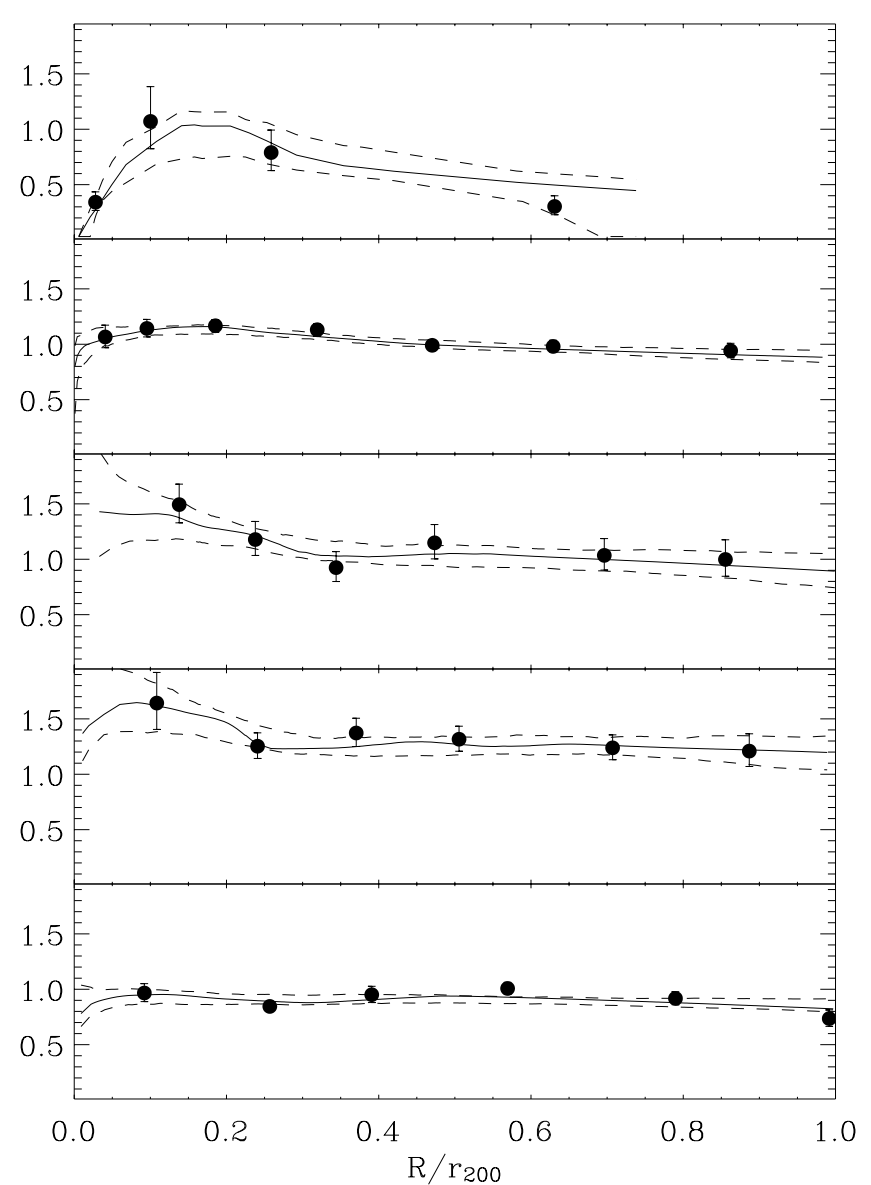

Fig. 2. The best LOWESS estimate (heavy line) of $\sigma_{p}(R)$, together with the $68 \%$ confidence levels (dashed lines), for each of the 5 galaxy classes, from top to bottom: $E_{\mathrm{br}}$, Early, $S_{\mathrm{e}}, S_{1}$, Subs. The filled circles with error bars indicate binned biweight estimates of $\sigma_{p}(R)$. The scale on the $y$-axis is in units of the global cluster velocity dispersion, calculated for all galaxies irrespective of type.

To our knowledge, this is the first time that the numberdensity and velocity-dispersion profiles for these 5 cluster galaxy classes have been derived with such accuracy and in such detail. Therefore, we briefly comment on the qualitative nature of the different $I(R), v(r)$ and $\sigma_{p}(R)$ before proceeding with the analysis.

Among galaxies outside substructure, the $E_{\mathrm{br}}$ have the steepest density profile in the centre, followed by the Early, the $S_{\mathrm{e}}$, and the $S_{1}$. This is a clear manifestation of the morphology-density relation (e.g. Dressler 1980), and of luminosity segregation (e.g. Rood \& Turnrose 1968 and Paper XI). Interestingly, the density profiles of both $S_{\mathrm{e}}$ and $S_{1}$ decrease towards the cluster centre, a clear indication that these galaxies avoid the central cluster regions. On the contrary, $E_{\mathrm{br}}$ are mostly found in the central cluster regions.

The Subs galaxies have a number-density profile that is rather steep in the centre, but shows a weak "plateau" at $\sim 0.6 r_{200}$. Note that the number density profile of this galaxy class could, in principle, be biased by systematic effects due to the selection procedure of the members of substructures, which might result in a radius-dependent detection efficiency. A comparison of the de-projected number densities of the Subs-class galaxies and of the bulk of the galaxies outside substructures, viz. the Early-class galaxies (right-hand panels of Fig. 1), shows that, within $\sim 0.6 r_{200}$, the two profiles have essentially identical logarithmic slopes. Beyond $\sim 0.6 r_{200}$ the number-density profile of the Subs galaxies is quite a bit flatter than that of the Early galaxies, until it steepens again beyond $\sim 1.0 r_{200}$. This was already noted in Paper XI. A comparison of the number-density profile of the Subs galaxies with that obtained by De Lucia et al. (2004) from their numerical models of substructures in cold dark matter haloes gives a similar result. The logarithmic slope between $0.1 r_{200}$ and $0.8 r_{200}$ of the number-density of haloes with masses $\sim 10^{13} M_{\odot}$ is about -1.6 , not very different from that of the Subs galaxies which is -1.5 .

The velocity dispersion of the $E_{\mathrm{br}}$ strongly decreases towards the centre, with a slower but equally large decrease outwards (remember that all velocity dispersions are normalized by the same, global velocity dispersion calculated for all galaxies irrespective of type). The special formation history and location of the $E_{\mathrm{br}}$ at the bottom of the cluster potential well is reflected in their very low central velocity dispersion. In contrast, galaxies of the Early class have a rather flat velocity-dispersion profile, changing by only $\approx \pm 20 \%$ over the virial region. The velocity-dispersion profiles of $S_{\mathrm{e}}$ and $S_{1}$ are rather similar, starting at high values near the centre with a fairly rapid decrease out to $r \approx 0.3 r_{200}$, and flattening towards larger projected distances. Yet, the velocity dispersion of the $S_{1}$ is larger than that of the $S_{\mathrm{e}}$ (and, in fact, of any other class) at all radii. It is perhaps interesting to note that the velocity-dispersion profiles of $S_{\mathrm{e}}$ and $S_{1}$ are remarkably similar to those of, respectively, the "backsplash" and infalling populations of subhaloes found in the numerical simulations of Gill et al. (2004).

Finally, the velocity-dispersion profile of the Subs class is very "cold" and flat, even flatter and "colder" than that of the Early class. One might wonder if this is due to the procedure by which the galaxies of the Subs class were selected, but it is very unlikely that the velocity dispersion of the Subs class is biased low by the selection. If anything, the actual velocity dispersion of the subclusters is overestimated because the internal velocity dispersion of the subclusters has not been corrected for. In Sect. 6.4 we discuss several estimates for the real velocity-dispersion profile, i.e. corrected for internal velocity dispersion and possible bias due to the selection.

\section{The mass profile}

In addition to the observed $I(R)-, v(r)$ - and $\sigma_{p}(R)$-profiles presented in Sect. 3 we also need an estimate of the mass profile $M(<r)$ for a determination of the $\beta(r)$-profiles. The mass profile that we will use here is the one that was derived in Paper XII, from the number density and velocity-dispersion profiles of the Early-class galaxies. As discussed in detail in Paper XII, the Early-class galaxies are likely to be in equilibrium with the cluster potential, as the formation of most of them probably antedates their entry into the cluster, so that they have had ample time to settle in the potential. In Paper XII we also showed that galaxies of the Early class have a nearly isotropic velocity distribution; this follows from an analysis of the shape of the distribution of their line-of-sight velocities. More specifically, assuming a constant velocity anisotropy for the 
Early-class galaxies, in Paper XII we concluded that $-0.6 \lesssim$ $\beta \lesssim 0.1$, where

$\beta(r) \equiv 1-\frac{\left\langle v_{\mathrm{t}}^{2}\right\rangle(r)}{\left\langle v_{\mathrm{r}}^{2}\right\rangle(r)}$,

and $\left\langle v_{\mathrm{r}}^{2}\right\rangle(r),\left\langle v_{\mathrm{t}}^{2}\right\rangle(r)$ are the mean squared components of the radial and tangential velocity (see, e.g., Binney \& Tremaine 1987). In this paper, we will often use the parameter $\beta^{\prime}$ instead of $\beta$ to describe the velocity anisotropy, where $\beta^{\prime}$ is defined as follows:

$\beta^{\prime} \equiv\left(\left\langle v_{\mathrm{r}}^{2}\right\rangle /\left\langle v_{\mathrm{t}}^{2}\right\rangle\right)^{1 / 2} \equiv(1-\beta)^{-1 / 2}$.

The constraint that we derived in Paper XII for $\beta$ of the Earlyclass galaxies translates into $\beta^{\prime} \simeq 1.0_{-0.2}^{+0.05}$.

For an isotropic velocity distribution $\left(\beta^{\prime}=1.0\right.$, or $\left.\beta=0\right)$ the mass profile follows from the isotropic Jeans equation:

$M(<r)=-\frac{r\left\langle v_{\mathrm{r}}^{2}\right\rangle}{G}\left(\frac{\mathrm{d} \ln v}{\mathrm{~d} \ln r}+\frac{\mathrm{d} \ln \left\langle v_{\mathrm{r}}^{2}\right\rangle}{\mathrm{d} \ln r}\right)$,

where $\left\langle v_{\mathrm{r}}^{2}\right\rangle(r)$ follows from:

$\left\langle v_{\mathrm{r}}^{2}\right\rangle(r)=-\frac{1}{\pi v(r)} \int_{r}^{\infty} \frac{\mathrm{d}\left[I(R) \times \sigma_{\mathrm{p}}^{2}(R)\right]}{\mathrm{d} R} \frac{\mathrm{d} R}{\sqrt{R^{2}-r^{2}}}$.

As with the de-projection of $I(R)$, Eq. (5) requires extrapolation of $\sigma_{p}(R)$ to the tidal radius (for details, see Appendix B.2 in Paper XII).

The resulting $M(<r)$, and its derivative $\rho(r)$ are shown in Fig. 4 of Paper XII. They are very well represented by a NFW profile (Navarro et al. 1997) with a scaling radius $r_{s}=0.25_{-0.10}^{+0.15} r_{200}$.

\section{The $S^{2}$ method for the solution of $\beta^{\prime}(r)$}

Binney \& Mamon (1982) were the first to show that it is possible to derive $\beta(r)$ when $I(R), \sigma_{p}(R)$ and $M(<r)$ are known. $\mathrm{S}^{2}$ gave a practical recipe for application of the method, and we give a brief summary of their method to determine the velocityanisotropy profile $\beta(r)$ for a given class of galaxies in equilibrium in a cluster gravitational potential with mass profile $M(<r)$ (note that in this context we use $\beta$ instead of $\beta^{\prime}$ to be consistent with the earlier papers).

The estimate of the mass profile $M(<r)$ is used together with the estimate of the $3 \mathrm{D}$ number density $v(r)$ (derived from $I(R)$ as before, see Eq. (1)), to calculate $\Psi(r)=-G M(<r) v(r) / r^{2}$. The observed functions $\sigma_{p}(R)$ and $I(R)$ are used to derive $H(R)=\frac{1}{2} I(R) \sigma_{p}^{2}(R)$, which in turn is used to calculate the function $K(r)$ by the Abel integral:

$K(r)=2 \int_{r}^{\infty} H(x) \frac{x \mathrm{~d} x}{\sqrt{x^{2}-r^{2}}}$.

Using the functions $\Psi(r)$ and $K(r)$, one obtains the following two equations for $\left\langle v_{\mathrm{r}}^{2}\right\rangle(r)$ and $\beta(r)$ :

$[3-2 \beta(r)] \times\left\langle v_{\mathrm{r}}^{2}\right\rangle(r)=\frac{-1}{v(r)} \int_{r}^{\infty} \Psi(x) \mathrm{d} x-\frac{2}{\pi r v(r)} \frac{\mathrm{d} K(r)}{\mathrm{d} r}$ and

$$
\begin{aligned}
\beta(r)\left\langle v_{\mathrm{r}}^{2}\right\rangle(r)= & \frac{1}{v(r) r^{3}} \int_{0}^{r} x^{3} \Psi(x) \mathrm{d} x \\
& +\frac{1}{\pi r v(r)} \frac{\mathrm{d} K(r)}{\mathrm{d} r} \\
& -\frac{3 K(r)}{\pi r^{2} v(r)}+\frac{3}{\pi r^{3} v(r)} \int_{0}^{r} K(x) \mathrm{d} x
\end{aligned}
$$

from which $\left\langle v_{\mathrm{r}}^{2}\right\rangle(r)$ and $\beta(r)$ can be derived.

The practical application of the method is far from trivial. First, one needs a smooth representation of the mass profile, which can be extrapolated confidently to large radii where we have not measured it. The extrapolation is done by using analytic mass profiles that adequately fit the $M(<r)$, such as the NFW profile (see Paper XII). This ensures that the integral of $\Psi(r)$ in Eq. (8) (whose upper integration limit we set to $6.67 r_{200}$; see Sect. 3) is not problematic. Fortunately, $\Psi(r)$ (which is negative) asymptotically approaches 0 with increasing $r$, and it does so with a sufficiently flat slope that the exact choice of the upper integration limit and the analytic representation of $M(<r)$ used for the extrapolation, do not influence the integral of $\Psi(r)$ in a significant way.

Secondly, one needs to extrapolate the observed velocitydispersion profiles, without having very strong constraints. For each class, we check that different (plausible) extrapolations have no significant effect on the results of the $S^{2}$ procedure within the observed radial range.

A third important point is that Eq. (8) contains two integrals which have a lower integration limit of $r=0$. Because it is quite difficult to determine the two integrands $\left(r^{3} \Psi(r)\right.$ and $\left.K(r)\right)$ at very small $r$ from observations, a plausible interpolation of $r^{3} \Psi(r)$ and $K(r)$ from the innermost measured 'point' to $r=0$ (for which both $r^{3} \Psi(r)$ and $K(r)$ are known from first principles) is needed. We made a special effort to ensure plausible interpolations from the innermost point for which the data is available to $r=0$, using low-order polynomials.

It will not come as a surprise, given the equations involved, that it is practically impossible to give estimates of the formal errors in $\beta^{\prime}(r)$ as derived with the $\mathrm{S}^{2}$ method. Approximate confidence levels on the $\beta^{\prime}(r)$ of each galaxy class were therefore determined by estimating the rms of four $\beta^{\prime}(r)$, obtained by applying the $S^{2}$ method to four subsamples, each half the size of the original sample. The fact that each subsample only contains half the number of galaxies in the original sample, is likely to compensate for the fact that the four subsamples are not all mutually independent, which could lead to underestimation of the true confidence levels.

We checked the robustness of our implementation of the $S^{2}$ method as follows. We applied the $S^{2}$ method to the galaxies of the Early class, adopting the mass profile that was determined using the same galaxies as isotropic tracers (see Paper XII and Sect. 4). Clearly, one should obtain $\beta(r) \equiv 0$, or, equivalently, $\beta^{\prime}(r) \equiv 1$ (see Eqs. (2) and (3)). The result is shown in Fig. 3. The shaded region indicates approximate 1- $\sigma$ confidence levels, derived as described above. Indeed, we find a velocity anisotropy very close to zero with $0.85 \leq \beta^{\prime}(r) \leq 1.15$ over the radial range $0 \leq r / r_{200} \leq 1.5$. Deviation from $\beta^{\prime}(r) \equiv 1$ 


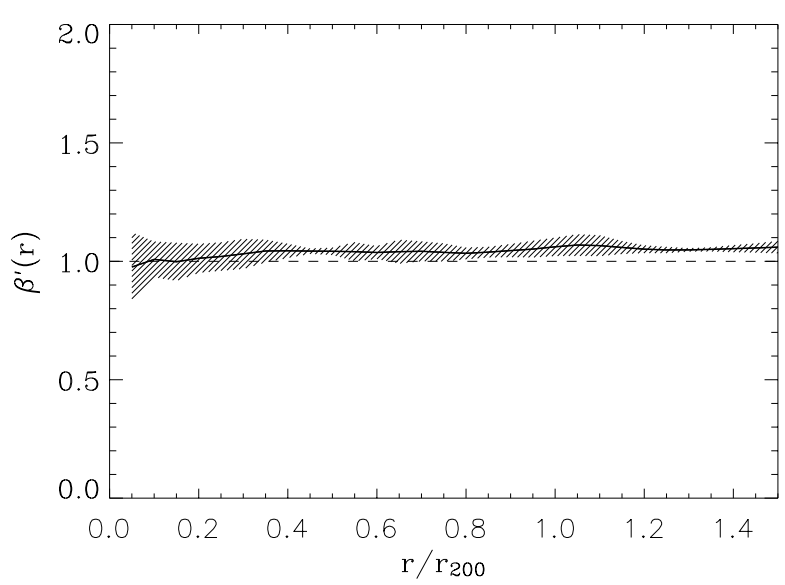

Fig. 3. The velocity anisotropy profile $\beta^{\prime}(r) \equiv\left\langle v_{\mathrm{r}}^{2}\right\rangle^{1 / 2} /\left\langle v_{\mathrm{t}}^{2}\right\rangle^{1 / 2}$, as derived for the galaxies of the Early class, using the mass profile that was derived assuming that the same galaxies have $\beta^{\prime}(r) \equiv 1$. The shaded region indicates approximate $1-\sigma$ confidence levels, as obtained by considering subsamples half the size of the original sample. The value of $\beta^{\prime}(r)$ is indeed quite close to 1 , as it should.

for the Early-class galaxies must be due to systematic errors arising from extrapolation uncertainties, and numerical noise in the inversion procedure (remember that our profiles are not analytic). Yet, the result in Fig. 3 indicates that our implementation of the $\mathrm{S}^{2}$ "inversion" works quite well.

We also applied a consistency test to all solutions that we obtained with the $\mathrm{S}^{2}$ method. I.e., we used the velocityanisotropy profile $\beta(r)$ obtained by the $\mathrm{S}^{2}$ method for a given galaxy class, to determine the projected velocity dispersion profile through (see, e.g., van der Marel 1994):

$v(r)\left\langle v_{\mathrm{r}}{ }^{2}\right\rangle(r)=-G \int_{r}^{\infty} \frac{v(\xi) M(<\xi)}{\xi^{2}} \exp \left[2 \int_{r}^{\xi} \frac{\beta \mathrm{d} x}{x}\right] \mathrm{d} \xi$

and

$I(R) \sigma_{p}^{2}(R)=2 \int_{R}^{\infty}\left(1-\beta(r) \frac{R^{2}}{r^{2}}\right) \frac{v r\left\langle v_{\mathrm{r}}^{2}\right\rangle(r) \mathrm{d} r}{\sqrt{r^{2}-R^{2}}}$.

We then compared this predicted velocity-dispersion profile with the observed $\sigma_{p}(R)$. In other words, we closed the loop, from observables and the mass profile to $\beta(r)$, then from $\beta(r)$ and the mass profile back to the observables.

The observed and predicted $\sigma_{p}(R)$ are always in very good agreement (see Sect. 6), despite the fact that we cannot determine $\beta(r)$ beyond $\sim 1.5 r_{200}$, while knowledge of this function to very large radii is required to solve Eq. (10). The behaviour of $\beta(r)$ at large radii is not important since the number-density profiles of all galaxy classes drop sufficiently fast with radius. Even for the $S_{1}$, which have the shallower $v(r)$, the effect of adopting two very different extrapolations of $\beta(r)$ to large radii (one derived from the analytical model proposed by Łokas $\&$ Mamon 2001, the other from the numerical simulations of Diaferio 1999) results in a $\lesssim 10 \%$ variation at any point of the predicted $\sigma_{p}(R)$.

\section{The velocity-anisotropy profiles}

We now investigate the orbits of the four classes of cluster galaxies that were not used to determine the mass profile,

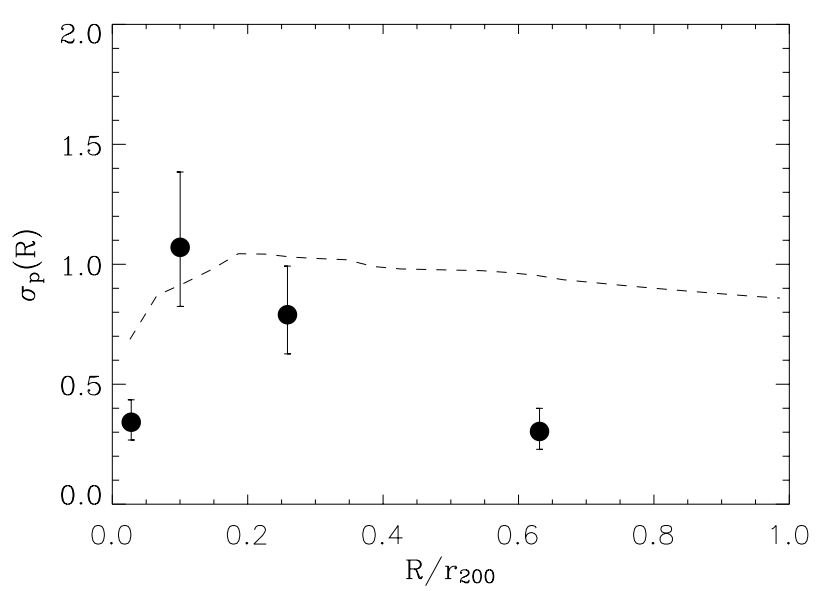

Fig. 4. The observed velocity-dispersion profile $\sigma_{p}(R)$ of the $E_{\mathrm{br}}$ galaxy class (dots with $1 \sigma$ errors), compared with the predicted $\sigma_{p}(R)$ (dashed line), assuming isotropic orbits in the gravitational potential determined from galaxies of the Early class.

viz. $E_{\mathrm{br}}, S_{\mathrm{e}}, S_{1}$, and Subs, in the gravitational potential determined using the galaxies of the Early class. In other words: we try to construct equilibrium solutions for each of the galaxy classes, with physically acceptable velocity-anisotropy profiles. However, first we try to find solutions with isotropic orbits (or, $\beta^{\prime} \equiv 1$ ). For this we need to solve Eq. (9), using the $v(r)$ of each class, and the mass profile $M(<r)$ as determined using the Early-class galaxies, setting $\beta^{\prime}(r) \equiv 1$. If the comparison between the predicted and the observed velocity-dispersion profile yields an acceptable $\chi^{2}$, we conclude that the galaxies of the given class can be considered isotropic tracers of the cluster gravitational potential.

After trying the isotropic solution, we then use the $S^{2}$ method to solve for $\beta^{\prime}(r)$. Note that, unlike Carlberg et al. (1997b,c), van der Marel et al. (2000), and Rines et al. (2003) we do not prescribe a functional form for $\beta^{\prime}(r)$, nor do we assume a constant value for $\beta^{\prime}(r)$.

\subsection{The brightest ellipticals}

The velocity-dispersion profile predicted for the $E_{\mathrm{br}}$ class assuming isotropic orbits is much flatter than the observed $\sigma_{p}(R)$ (see Fig. 4). We can reject the isotropic solution at $>99 \%$ confidence level ( $\chi^{2}=98$ on 4 data-points).

Interestingly, abandoning the isotropy assumption does not help. I.e. there is no physical solution for which the $E_{\mathrm{br}}$ are in equilibrium in the cluster gravitational potential (i.e. the $\mathrm{S}^{2}$ method predicts negative $\left\langle v_{\mathrm{r}}^{2}\right\rangle$ and $\beta(r)>1$ over most of the radial range covered by our observations). There are two straightforward interpretations of this result: either the galaxies of the $E_{\mathrm{br}}$ class are indeed out of dynamical equilibrium, or they do not fulfil the conditions for the application of the Jeans equation. We will return to this point in Sect. 7.

\subsection{The early spirals}

For the galaxies of the $S_{\mathrm{e}}$ class we do find acceptable equilibrium solutions assuming an isotropic velocity distribution. 


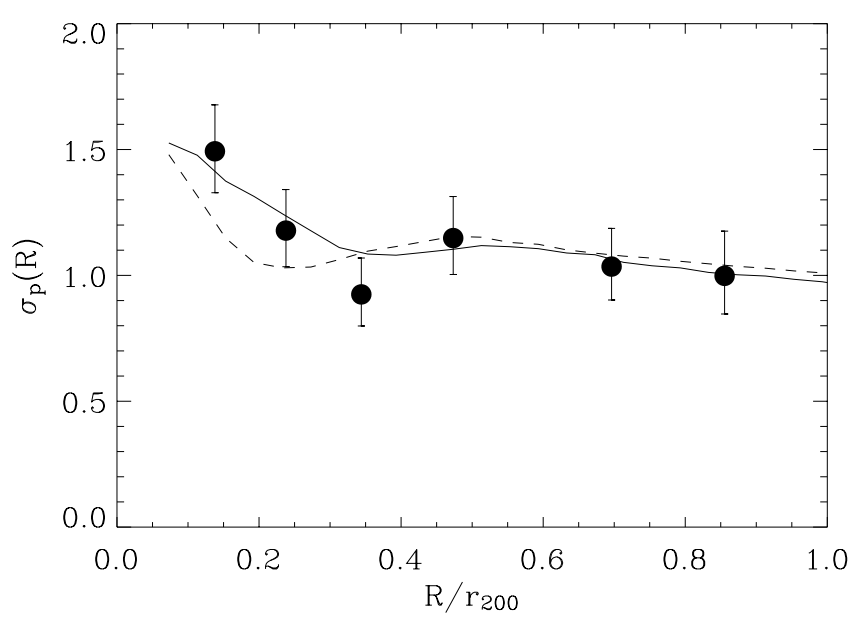

Fig. 5. The observed velocity-dispersion profile $\sigma_{p}(R)$ of the $S_{\mathrm{e}}$ galaxy class (dots with $1 \sigma$ errors), compared with the predicted $\sigma_{p}(R)$ (dashed line), obtained by assuming isotropic orbits in the gravitational potential determined from galaxies of the Early class, and with the predicted $\sigma_{p}(R)$ (solid line), obtained by using the velocity-anisotropy profile $\beta^{\prime}(r)$ determined by the $S^{2}$ method and shown in Fig. 6.

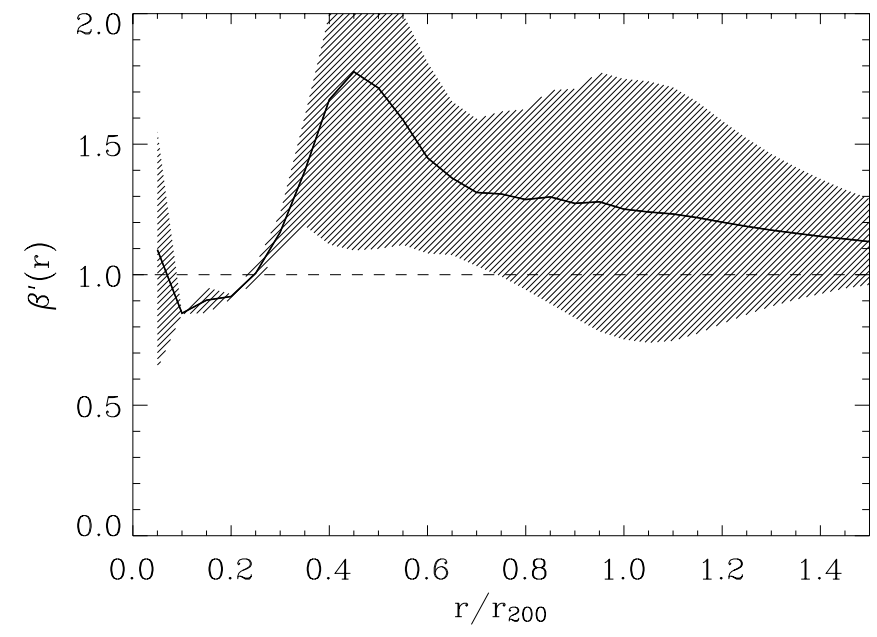

Fig. 6. The anisotropy profile, $\beta^{\prime}(r) \equiv\left[\left\langle v_{\mathrm{r}}^{2}\right\rangle /\left\langle v_{\mathrm{t}}^{2}\right\rangle\right]^{1 / 2}$ as derived for the galaxies of the $S_{\mathrm{e}}$ class, through the $\mathrm{S}^{2}$ method. The shaded region indicates approximate $1-\sigma$ confidence levels, as obtained by considering subsamples half the size of the original sample.

The predicted velocity dispersion profile provides an acceptable fit to the observed $\sigma_{p}(R)\left(\chi^{2}=5.2\right.$ on 6 data-points, rejection probability of $61 \%$ ). This profile is shown as a dashed line in Fig. 5, together with the observations and their $1 \sigma$ errors. Note that, although the data can be represented satisfactorily with isotropic orbits in the mass profile determined using the Early-class galaxies, the innermost values of $\sigma_{p}(R)$ are somewhat underpredicted.

The velocity-anisotropy profile of the $S_{\mathrm{e}}$ class (determined via the $S^{2}$ method) is shown as the solid line in Fig. 6 . The velocity-anisotropy profile $\beta^{\prime}(r)$ is very close to unity near the centre, then rises to a maximum value of $\approx 1.8$ at $r \approx 0.45 r_{200}$ and then decreases again to reach $\approx 1.1$ at $r / r_{200} \approx 1.5$. As mentioned before, we checked the quality of this $\beta^{\prime}(r)$ solution by calculating the implied velocity-dispersion profile, solving

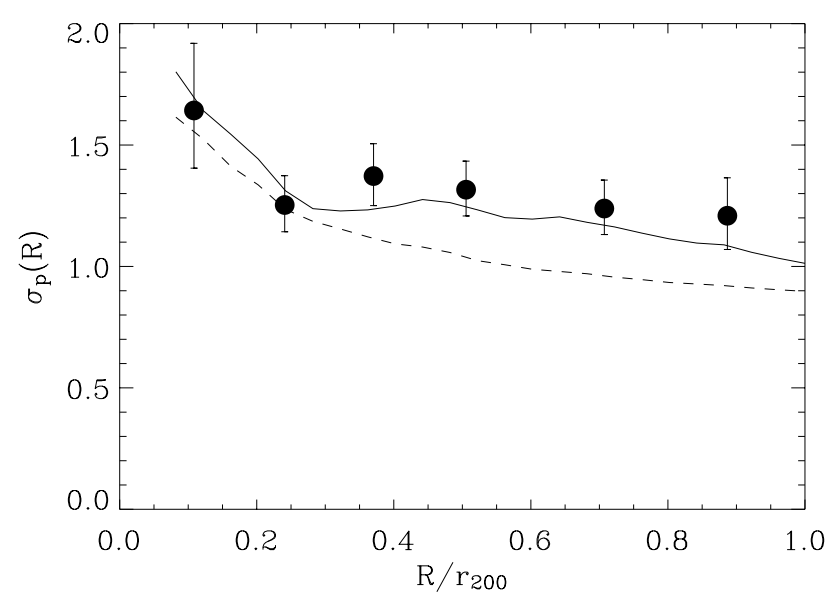

Fig. 7. The observed velocity-dispersion profile $\sigma_{p}(R)$ of the $S_{1}$ galaxy class (dots with $1 \sigma$ errors), compared with the predicted $\sigma_{p}(R)$ (dashed line), obtained by assuming isotropic orbits in the gravitational potential determined from galaxies of the Early class, and with the predicted $\sigma_{p}(R)$ (solid line), obtained by using the velocityanisotropy profile $\beta^{\prime}(r)$ determined by the $S^{2}$ method and shown in Fig. 8.

Eq. (9) for this $\beta(r)$. The $\sigma_{p}(R)$ predicted in this way from the $\beta^{\prime}(r)$ indicated by the solid line in Fig. 6, is shown in Fig. 5, also as a solid line. As expected, the latter is closer $\left(\chi^{2}=2.0\right.$ on 6 data-points, rejection probability of $16 \%)$ to the observations than the isotropic solution (dashed line) but not significantly so, because the isotropic model already yields an acceptable fit to the data. As a matter of fact, the uncertainties on the $\beta^{\prime}(r)$ profile determined via the $S^{2}$ method are quite large, so that any deviation from the isotropic solution is not really significant.

\subsection{The late spirals $+E L G$}

For the galaxies of the $S_{1}$ class we do not find acceptable equilibrium solutions assuming an isotropic velocity distribution. This is illustrated in Fig. 7, where the predicted $\sigma_{p}(R)$ (dashed line) is clearly seen to provide a poor fit to the data $\left(\chi^{2}=18.2\right.$ on 6 data-points, rejection probability $\left.>99 \%\right)$. Beyond $R>0.3 r_{200}$ the predicted velocity-dispersion profile is well below the observed values. Hence, purely isotropic orbits are rejected.

We then considered anisotropic solutions. The velocityanisotropy profile of the $S_{1}$ class (determined via the $\mathrm{S}^{2}$ method) is shown in Fig. 8. The profile is very close to unity out to $r \approx 0.7 r_{200}$, where it starts growing almost linearly with radius to reach a value of $\approx 1.8$ at $r / r_{200} \approx 1.5$. As usual, we checked the quality of the $\beta^{\prime}(r)$-solution by calculating the implied velocity-dispersion profile, solving Eq. (9) for this $\beta(r)$. The $\sigma_{p}(R)$ predicted in this way from the velocity-anisotropy profile indicated in Fig. 8, is shown in Fig. 7 as a solid line. As expected, it reproduces quite well the observed $\sigma_{p}(R)$ of $S_{1}$.

In the case of the $S_{1}$ class the velocity-dispersion profile predicted with the $\beta^{\prime}(r)$ obtained with the $S^{2}$ method not only fits the data better than the isotropic case (this is also true for the $S_{\mathrm{e}}$-class galaxies), but it also does so in a significantly better manner $\left(\chi^{2}=2.6\right.$ on 6 data-points, rejection 


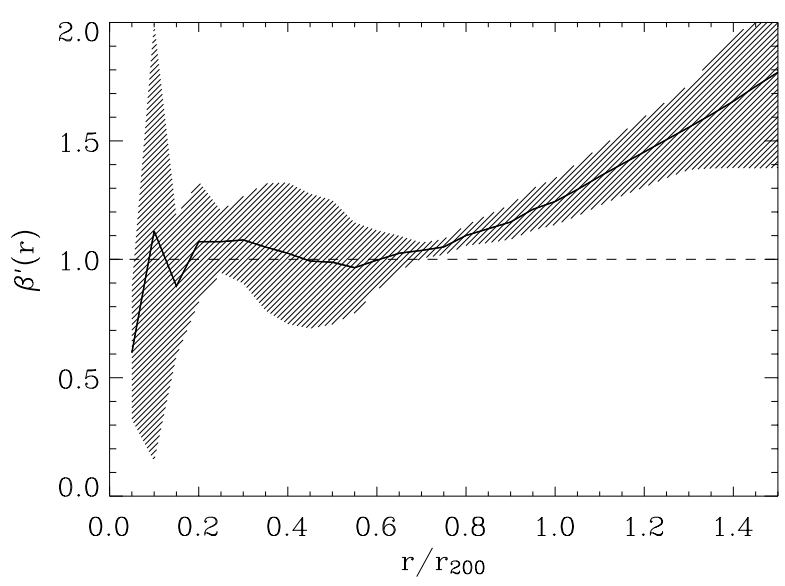

Fig. 8. The anisotropy profile, $\beta^{\prime}(r) \equiv\left[\left\langle v_{\mathrm{r}}^{2}\right\rangle \mid\left\langle v_{\mathrm{t}}^{2}\right\rangle\right]^{1 / 2}$ as derived for the galaxies of the $S_{1}$ class, through the $S^{2}$ method. The shaded region indicates approximate 1- $\sigma$ confidence levels, as obtained by considering subsamples half the size of the original sample.

probability $23 \%$ ). Therefore, mild radial anisotropy is needed in order to put the $S_{1}$-class galaxies in dynamical equlibrium in the cluster potential.

\subsection{The galaxies in substructures}

As for the $S_{1}$-class galaxies, we do not find acceptable equilibrium solutions for the galaxies of the Subs class if we assume an isotropic velocity distribution. As can be seen in Fig. 9, the predicted $\sigma_{p}(R)$ (dashed line) is way off the data (dots with error-bars; $\chi^{2}=118.5$ on 6 data-points, rejection probability $>99 \%$ ), and overestimates the observed velocity dispersion at essentially all radii.

Using the $S^{2}$ method for the Subs class, with the observed $\sigma_{p}(R)$ we obtain the $\beta^{\prime}(r)$ displayed as a solid line in Fig. 10. The orbits are tangentially anisotropic at all radii. As usual, we checked the $\beta^{\prime}(r)$ solution in the space of observables; the predicted $\sigma_{p}(R)$ is in excellent agreement with the observed one (see Fig. $9 ; \chi^{2}=5.1$ on 6 data-points, rejection probability $60 \%)$.

In the lower panel of Fig. 10, the shaded region indicates approximate $1-\sigma$ confidence interval, obtained as described before. However, in this case the real confidence interval is probably significantly larger, for two reasons.

First, in using the observed velocity-dispersion profile of the galaxies in subclusters, we have ignored the internal velocity dispersion of the subclusters. This means that the real velocity dispersion is smaller than the observed one. We will make several assumptions for the (possibly R-dependent) value of the apparent internal velocity dispersion of the subclusters. In Paper XI we estimated the internal velocity dispersion of the identified subclusters, and obtained a value of $\sim 400$ $500 \mathrm{~km} \mathrm{~s}^{-1}$, essentially independent of projected radius $R$. However, the true internal velocity dispersion of a subcluster is likely to be smaller, because the above estimate is biased high by galaxies that do not belong to the subcluster but have been wrongly assigned to it by the selection algorithm.

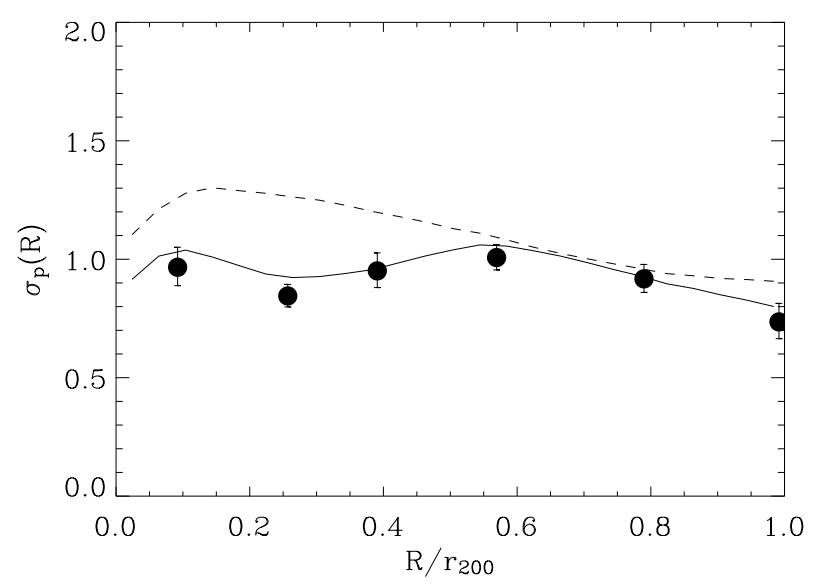

Fig. 9. The observed velocity-dispersion profile $\sigma_{p}(R)$ of the Subs galaxy class (dots with $1 \sigma$ errors), compared with the predicted $\sigma_{p}(R)$ (dashed line), obtained by assuming isotropic orbits in the gravitational potential determined from galaxies of the Early class, and with the predicted $\sigma_{p}(R)$ (solid line), obtained by using the velocityanisotropy profile $\beta^{\prime}(r)$ determined by the $S^{2}$ method and shown by the solid line in Fig. 10 (lower panel).

A more realistic estimate of a subcluster internal velocity dispersion is probably $250 \mathrm{~km} \mathrm{~s}^{-1}$, a value close to the average velocity dispersion of galaxy groups (see, e.g., Ramella et al. 1989). First, this constant value was subtracted in quadrature from the observed $\sigma_{p}(R)$ of the Subs class to produce the corrected velocity dispersion of subclusters shown as a dashed line in the upper panel of Fig. 10. From this corrected $\sigma_{p}(R)$, and using - as before - the observed $I(R)$, we derived the corrected version of $\beta^{\prime}(r)$, indicated by the dashed line in the lower panel of Fig. 10). This second solution implies even stronger tangential anisotropy of the velocity distribution, which is not surprising since a larger fraction of the (smaller) line-of-sight velocities is required to balance the same cluster potential.

However, it is possible that due to the selection procedure, or for physical reasons, we should not substract a constant value for the internal velocity dispersion of the subclusters. Therefore, we have assumed (rather arbitrarily) two different alternative solutions for the real velocity-dispersion profile of the Subs galaxies. These are shown as the dashed-dotted and dashed-triple-dotted curves in the upper panel of Fig. 10. The former assumes a larger bias in the observed velocity dispersion in the outer regions, while the latter mimics a larger bias in the central region. The important point in both assumptions is that the we must always deconvolve the observed $\sigma_{p}(R)$ with at least $250 \mathrm{~km} \mathrm{~s}^{-1}$ internal dispersion of the subclusters.

For both assumptions about the real velocity-dispersion profile of the Subs galaxies, we calculated $\beta^{\prime}(r)$, assuming as before - that the observed $I(R)$ is unbiased. The results are shown in the lower panel of Fig. 10, where the same coding is used as in the upper panel. Not surprisingly, the evidence for tangential anisotropy of the Subs galaxies does not go away; if anything it gets stronger (in the most extreme cases, no physical solution can be found beyond a certain radius).

However, before we can accept this conclusion to be robust, we must investigate the effect of possible biases in $I(R)$. Without real modelling, we have considered two fairly extreme 

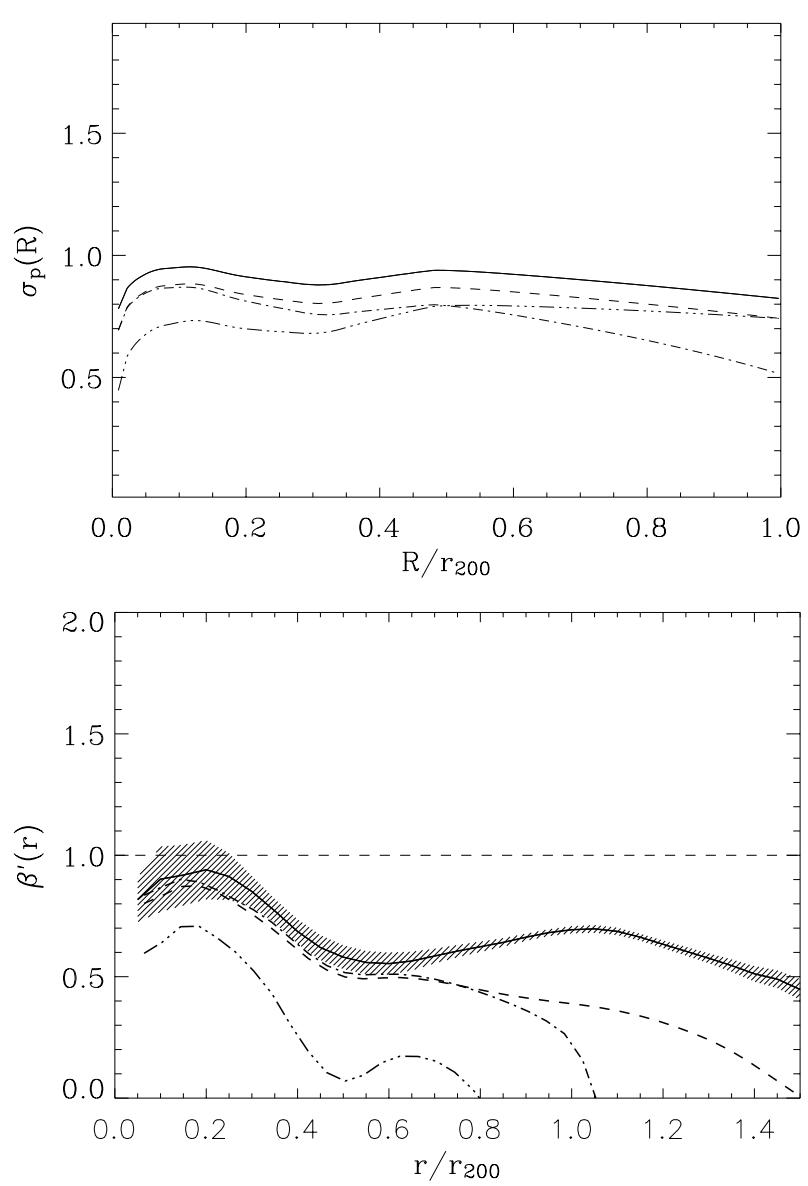

Fig. 10. Upper panel: the observed velocity-dispersion profile $\sigma_{p}(R)$ of the galaxies in subclusters (solid line). The dashed line shows the result of deconvolving $\sigma_{p}(R)$ with an assumed constant internal velocity dispersion of $250 \mathrm{~km} \mathrm{~s}^{-1}$. The two other curves assume radius-dependent internal velocity dispersions in the range 250 $450 \mathrm{~km} \mathrm{~s}^{-1}$. Lower panel: the anisotropy profiles, $\beta^{\prime}(r) \equiv\left[\left\langle v_{\mathrm{r}}^{2}\right\rangle /\left\langle v_{\mathrm{t}}^{2}\right\rangle\right]^{1 / 2}$ derived for the galaxies of the Subs class, through the $S^{2}$ method with the observed $I(R)$ and the four $\sigma_{p}(R)$-estimates shown in the upper panel (with identical coding). The shaded region indicates approximate 1- $\sigma$ confidence levels around the solution that uses the observed $\sigma_{p}(R)$-curve. These were obtained by considering subsamples half the size of the original sample.

possibilities. In the first one we assumed that the plateau around $0.6 r_{200}$ in the observed $v(r)$ of the Subs galaxies (see Fig. 1 and Sect. 3) is (at least partly) an artefact due to the selection procedure. Consequently, we multiplied $v(r)$ by a "constant" factor of 2 below $\sim 0.6 r_{200}$. The other possibility assumes that the logarithmic slope of $v(r)$ below $0.6 r_{200}$ is $\simeq 40 \%$ flatter than actually observed.

These two extreme assumptions about $v(r)$ were combined with the various assumed estimates of $\sigma_{p}(R)$ to solve for $\beta^{\prime}(r)$ of the Subs galaxies. It appears that the conclusion of tangential orbits is not affected by the different assumed shapes of $v(r)$ below $0.6 r_{200}$, and is thus primarily driven by the low values of the velocity dispersion of the Subs galaxies. In other words, we find that, even without a detailed modelling of the selection effects and a possible dependence of the internal velocity dispersion of the subclusters on radius, the conclusion about the tangential orbits is robust. The implications of this result will be discussed in Sect. 7.

\section{Discussion}

Adopting the mass profile as determined from the galaxies of the Early class, we searched for equilibrium solutions for the other four classes. As a first step, we assumed isotropy of the velocity distribution, but subsequently we also solved for the anisotropy profile $\beta^{\prime}(r)$ using the $S^{2}$ set of equations (see Sect. 5). For the $E_{\mathrm{br}}$ class, we could not obtain equilibrium solutions, no matter what we assumed for their velocityanisotropy profile. For the $S_{\mathrm{e}}$ class the isotropic solution was found to be quite acceptable. Yet, the velocity-anisotropy profile of the $S_{\mathrm{e}}$ class, as determined with the $\mathrm{S}^{2}$-method, shows a slight radial anisotropy at $r \approx 0.45 r_{200}$. For the $S_{1}$ class, the isotropic solution is rejected. Their velocity-anisotropy profile, determined with the $\mathrm{S}^{2}$ method, is close to zero out to $r \approx$ $0.7 r_{200}$, and then increases almost linearly outwards, reaching a radial anisotropy of $\beta^{\prime} \approx 1.8$ (corresponding to $\beta \approx 0.7$ ) at $r \approx 1.5 r_{200}$. For the Subs class the isotropic solution must also be rejected. Taken at face value, the data for this class imply substantial tangential anisotropy. However, this result may be affected by systematic effects related to the selection of Subs galaxies. Until these effects have been modelled in detail, the conclusion of tangential anisotropy must be considered with some caution.

Our conclusion that both early-type and late-type galaxies are in equilibrium in the cluster potential, with the latter on more radially-elongated orbits, is supported by several other studies in the literature. The larger velocity dispersion and/or the steeper velocity-dispersion profile of late-type galaxies with respect to early-type galaxies, have often been interpreted as evidence for infalling motions, and even for departure from virial equilibrium (Moss \& Dickens 1977; Sodré et al.1989; Paper III; Adami et al. 1998a; Solanes et al. 2001). However, Carlberg et al. (1997a) already pointed out that the latter does not need to be the case. They found that red and blue galaxies in the CNOC clusters are both in dynamical equilibrium in the cluster gravitational potential, but they were not able to constrain the velocity anisotropy of these galaxies.

From a more detailed analysis of the same dataset, van der Marel et al. (2000) were able to constrain the average velocity anisotropy (assumed to be constant) of all CNOC cluster galaxies, to $0.75 \leq \beta^{\prime} \leq 1.2$ (95.4\% c.l.). This is a similar to what we found for the Early galaxies in Paper XII, and from which we concluded that those have isotropic orbits. Mahdavi et al. (1999) showed that ELG in groups have an anisotropic velocity distribution, at the $95.4 \%$ c.l., with a best-fit constant $\beta^{\prime} \approx 1.8$, whereas absorption-line galaxies in groups have a best-fit constant $\beta^{\prime} \approx 1.4$, which is not, however, significantly different from unity. Both values seem somewhat higher than the values we find, which could indicate that the fraction of infalling galaxies is larger in groups than in clusters.

Biviano (2002), also using the ENACS dataset, concluded that, if absorption-line galaxies have zero anisotropy $\beta^{\prime}=1.0$, ELG have an average constant anisotropy of $1.3 \leq \beta^{\prime} \leq 1.6$ (68\% c.l.). This range is in reasonable agreement with our 
result for the velocity anisotropy profile of the $S_{1}$ galaxies (see Fig. 8), considering that many $S_{1}$ galaxies are found at large radii, where their radial velocity-anisotropy is largest. Natarajan \& Kneib (1996) concluded that galaxies in A2218 have tangential orbits in the central region, and radial outside, with an anisotropy profile resembling the one we find for $S_{1}$. Finally, Ramírez \& de Souza (1998) and Ramírez et al. (2000) concluded that early-type galaxies have more eccentric orbits than late-type galaxies, but their result arises from incorrect assumptions in their method, as discussed by van der Marel et al. (2000) and Biviano (2002).

The present analysis is the first to consider the orbits of 5 distinct classes of cluster galaxies. Using the Early class as a reference, we find that 3 of the remaining 4 classes are in dynamical equilibrium within the cluster gravitational potential; this is manifestly not the case for the $E_{\mathrm{br}}$. The most likely explanation for our failure to find solutions of the collisionless Jeans equation for the $E_{\mathrm{br}}$, is that the $E_{\mathrm{br}}$ either formed very near the cluster centre, or moved there by losing kinetic energy subject to dynamical friction. At the same time, they probably have grown through merging with other galaxies. These processes lead to a loss of the orbital energy of these galaxies. As a matter of fact, the very low velocity dispersion of the $E_{\mathrm{br}}$ at the cluster centre can be understood with the model of Menci \& Fusco-Femiano (1996), which is a solution of the collisional Boltzmann-Liouville equation, and hence accounts for galaxy collisions and merging processes.

The conclusion that the Early-class galaxies have a nearly isotropic velocity distribution is not surprising, given the large body of evidence indicating that ellipticals are an old cluster component. If they form and become part of the cluster before it virializes, they can obtain isotropic orbits through violent relaxation. From the distribution of the ratio $r_{\text {peri }} / r_{\text {apo }}$ of the dark matter halos in their simulations of rich clusters, Ghigna et al. (1998) concluded that about $25 \%$ of the halos are on orbits more radial than $1: 10$, where the median ratio is $1: 6$. Comparison with our result is not immediate, but of the galaxies outside substructures $36 \%$ belong to the $S_{\mathrm{e}}$ and $S_{1}$ classes. Since about two-thirds of those are late spirals or ELG, which are the galaxies showing most of the velocity anisotropy, these could indeed correspond to the halos with orbits more radial than $1: 10$ in the $r_{\text {peri }} / r_{\text {apo }}$-ratio.

The increase of the radial-velocity anisotropy with radius of the $S_{1}$ (see Fig. 8) is a feature commonly found in numerical simulations of dark matter haloes. E.g., the numerical simulations of Tormen et al. (1997) predict an increasing radial velocity anisotropy from $r / r_{200} \sim 0.3$ outwards, reaching $\beta^{\prime} \sim 1.8$ at $r / r_{200} \sim 1.5$, and the numerical simulations described by Diaferio et al. (2001) predict a similar, though somewhat more irregular, behaviour of the velocity anisotropy profile, with a maximum anisotropy of $\beta^{\prime} \sim 1.4$. This anisotropy profile results from infall motions of the field haloes into the cluster, and from the subsequent isotropization of the velocity distribution of these haloes as they move towards the denser cluster centre.

The similarity of the $\beta^{\prime}(r)$ of the $S_{1}$-class galaxies and of the dark matter haloes in the models is quite interesting and it probably means that the $S_{1}$ galaxies still retain memory of their infall motion from the field. The fact that a large fraction of the
$S_{1}$ have emission-lines indicates that they have not yet lost their gas as a consequence of tidal stripping, galaxy collisions, or ram pressure. Hence it is unlikely that the $S_{1}$ we observe have spent much time in the hostile cluster environment, and many of them could indeed even be on their first cluster crossing.

On the other hand, the small velocity anisotropy of $S_{1}$ near the centre probably reflects the fact that the galaxies we identify as $S_{1}$ must avoid, or have avoided, the central region. Those $S_{1}$ that have a significant radial anisotropy near the centre will cross the very dense central cluster regions, where they cannot survive and get disrupted, either to form dwarfs, or to contribute to the diffuse intra-cluster light (Moore et al. 1999). As a matter of fact, $S_{1}$ are not found in the cluster central regions (see Fig. 1 and Paper XI). The existence of faint spiral structures in some dwarf spheroidals has now been demonstrated (Jerjen et al. 2000; Barazza et al. 2002; Graham et al. 2003). Interestingly, Conselice et al. (2001) found that, in the Virgo cluster, the dwarf spheroidals have a velocity distribution more similar to that of the spirals, than to that of the ellipticals.

Our analysis shows that an acceptable equilibrium solution exists for $S_{\mathrm{e}}$ with zero velocity anisotropy. Hence, it is likely that these galaxies are not very recent arrivals, since there is no evidence for memory of their initial infall motion. These $S_{\mathrm{e}}$ galaxies are more likely to survive the hostile cluster environment than $S_{1}$, because of their higher surface brightness (see Paper X; Moore et al. 1999). This is consistent with the results of numerical simulations showing that clusters contain red disk galaxies that, after accretion from the field, attain dynamical equilibrium in 1-2 Gyr (Diaferio et al. 2001).

Additional indirect support for the scenario described above comes from the similarity of the $S_{\mathrm{e}}$ and the $S_{1}$ velocity dispersion profiles with those of, respectively, the "backsplash" subhaloes, and the subhaloes on first infall, identified by Gill et al. (2004) in their $N$-body simulations of galaxy clusters. This similarity suggests that many $S_{1}$ could be on first infall, while the $S_{\mathrm{e}}$ at large radii have already crossed the cluster core.

In Paper $\mathrm{X}$ it was argued that $S_{\mathrm{e}}$ are likely to be the progenitors of SOs. This conclusion is based on three different pieces of evidence: (1) the strong increase of SOs in clusters since $z \sim 0.5$ (Dressler et al. 1997; Fasano et al. 2000), accompanied by a similar decrease of the spiral fraction; (2) the morphologydensity relation (Thomas et al. 2004, hereafter Paper IX) which shows that the local projected density around $S_{\mathrm{e}}$ is smaller than around SOs; and (3) the strong similarity of the bulge luminosity of $S_{\mathrm{e}}$ and S0s (Paper X). If $S_{\mathrm{e}}$ transform into S0s and if the velocity distribution of S0s is isotropic (see Paper XII), it is only natural that the velocity distribution of $S_{\mathrm{e}}$ is also isotropic. Otherwise, the timescale of the morphological-transformation process should be similar to that of the velocity isotropization.

Even if the isotropic solution is perfectly acceptable for the $S_{\mathrm{e}}$, the data, when taken at face value, imply some radial velocity anisotropy. Although the significance of the anisotropy is rather low, we are tempted to speculate about a possible cause, if the anisotropy at $r / r_{200} \approx 0.45$ were real. Galaxies with radial velocity-anisotropy, moving on radially elongated orbits, will move relatively fast near the cluster centre. It is possible, if not likely, that the high radial velocity is a necessary condition 
for $S_{\mathrm{e}}$ to avoid impulsive encounters and thus transformation into S0s in the central high-density cluster region.

Finally, galaxies in substructures provide an intrigueing view into the processes that are important in the formation of clusters. Recently, several groups have studied the properties of substructures within dark-matter haloes over a range of total masses that includes those of rich clusters (e.g. De Lucia et al. 2004; Taylor \& Babul 2004). We already mentioned the agreement between our radial number-density profile of Subs galaxies and the radial distribution of $10^{13} M_{\odot}$ substructures in the models of De Lucia et al. (2004, see Sect. 3). Those models also show that the more massive substructures are preferentially located in the external regions of their parent haloes. This is most likely due to tidal truncation and stripping of substructures that reach the dense central regions. In addition, orbital decay can also contribute to this mass segregation (e.g. Tormen et al. 1998). The apparent paucity of Subs galaxies in the inner regions of our clusters may thus well be the result of mass segregation, instead of selection bias.

Taylor \& Babul (2004) discuss the evolution of the orbits of the infalling substructures, and they conclude that disruption occurs sooner for more radial orbits. This will lead to a tangentially anisotropic distribution of orbits of the surviving substructures, which is exactly what we find. So, even if the details of the tangential anisotropy of the Subs galaxies requires additional modelling, the result itself appears robust and not unexpected or implausible.

\section{Summary and conclusions}

We determined the equilibrium solutions for galaxies of the 4 classes that were not used as tracers of the cluster potential. For this, we solved the inverse Jeans equation, using the method of $S^{2}$. We found equilibrium solutions for galaxies of the $S_{\mathrm{e}}$, the $S_{1}$, as well as the Subs classes, but not for galaxies of the $E_{\mathrm{br}}$-class. The equilibrium solution found for galaxies of the $S_{\mathrm{e}}$ class was found to be consistent with them being on isotropic orbits, except perhaps just outside the cluster central region. On the other hand, isotropic solutions were found not to be acceptable for galaxies of either the $S_{1}$ or the Subs classes. Galaxies of the $S_{1}$ class were found to be on mildly radial orbits, with the radial velocity-anisotropy increasing outwards. On the contrary, tangential orbits seem to characterize galaxies of the Subs class, but the significance of this result is difficult to assess in view of possible systematics effects we have considered.

Our results support hierarchical models for the build-up of galaxy clusters (see also Paper XII). Our results also constrain the evolutionary history of cluster galaxies. They are consistent with, if not suggestive of, a scenario where the very bright ellipticals form very early, and sink to the bottom of the still forming cluster potential well, losing orbital energy. In our scenario, the less bright ellipticals, together with the SOs (the Early-class galaxies), were already part of the cluster at the epoch of its formation, and developed isotropic orbits through the process of violent relaxation, or have lived sufficiently long in the cluster to have lost any memory of original radial infall motions, through isotropization of their orbits.
This is probably also the case for the early spirals, which make them acceptable candidates for being the progenitors of S0s, also in view of their structural properties. We speculate that some early spirals near the cluster centre have managed to escape transformation into S0s as a result of a selection effect in the velocity distribution. Finally, many late spirals and emission-line galaxies (excluding those of early morphology) are likely to be field galaxies recently arrived into the cluster. Their radial infall motions are gradually isotropized as they approach the cluster centre, until they get disrupted or transformed into dwarf spheroidals as a consequence of collisions and, in particular, tidal effects.

The galaxies in substructures apparently avoid the central regions and they appear to be on tangential orbits. Although some modelling remains to be done to assess the details of the implied anisotropy profile, the conclusion of tangential anisotropy appears to be robust. Interestingly, both effects are also seen in numerical simulations, and they result from the mechanisms that "destroy" the substructures as they get nearer to the cluster cores.

Acknowledgements. We thank Gary Mamon, Alain Mazure, and Tom Thomas for useful discussions. A.B. acknowledges the hospitality of Leiden Observatory. P.K. acknowledges the hospitality of Trieste Observatory. This research was partially supported by the Italian Ministry of Education, University, and Research (MIUR grant COFIN2001028932 "Clusters and groups of galaxies, the interplay of dark and baryonic matter"), and by the LeidsKerkhoven-Bosscha Fonds.

\section{References}

Adami, C., Biviano, A., \& Mazure, A. 1998a, A\&A, 331, 439

Adami, C., Mazure, A., Biviano, A., Katgert, P., \& Rhee, G. 1998b, A\&A, 331, 493 (Paper IV)

Barazza, F. D., Binggeli, B., \& Jerjen, H. 2002, A\&A, 391, 823

Beers, T. C., Flynn, K., \& Gebhardt, K. 1990, AJ, 100, 32

Beisbart, C., Valdarnini, R., \& Buchert, T. 2001, A\&A, 379, 412

Binney, J., \& Mamon, G. 1982, MNRAS, 200, 361

Binney, J., \& Tremaine, S. 1987, Galactic Dynamics (Princeton: Princeton University Press)

Biviano, A. 2002, in Tracing Cosmic Evolution with Galaxy Clusters, ed. S. Borgani, M. Mezzetti, \& R. Valdarnini, 127

Biviano, A., \& Katgert, P. 2003, Ap\&SS, 285, 25

Biviano, A., Girardi, M., Giuricin, G., Mardirossian, F., \& Mezzetti, M. 1992, ApJ, 396, 35

Biviano, A., Durret, F., Gerbal, D., et al. 1996, ApJ, 311, 95

Biviano, A., Katgert, P., Mazure, A., et al. 1997, A\&A, 321, 84 (Paper III)

Biviano, A., Katgert, P., Thomas, T., Mazure, A., \& Adami, C. 1999, 1st workshop of the Italian network Formazione ed evoluzione delle galassie, ed. C. Chiosi, L. Portinari, \& R. Tantalo, 45

Biviano, A., Katgert, P., Thomas, T., \& Adami, C. 2002, A\&A, 387, 8 (Paper XI)

Biviano, A., Katgert, P., Thomas, T., \& Mazure, A. 2003, Rev. Mex. Astron. Astrofis. (Series de Conferencias), 17, 191

Biviano, A., Katgert, P., \& Mazure, A. 2004, in Clusters of Galaxies: Probes of Cosmological Structure and Galaxy Evolution, from the Carnegie Observatories Centennial Symposia, 2004, ed. J. S. Mulchaey, A. Dressler, \& A. Oemler (Pasadena: Carnegie Observatories) 
Carlberg, R. G., Yee, H. K. C., Ellingson, E., et al. 1997a, ApJ, 476, L7

Carlberg, R. G., Yee, H. K. C., \& Ellingson, E. 1997b, ApJ, 478, 462

Carlberg, R. G., Yee, H. K. C., Ellingson, E., et al. 1997c, ApJ, 485, L13

Colless, M., \& Dunn, A. M. 1996, ApJ, 458, 435

Conselice, C. J., Gallagher, J. S, \& Wyse, R. F. G. 2001, ApJ, 559, 791

De Lucia, G., Kauffman, G., Springel, V., et al. 2004, MNRAS, 348, 333

den Hartog, R., \& Katgert, P. 1996, MNRAS, 279, 349

de Theije, P. A. M., \& Katgert, P. 1999, A\&A, 341, 371 (Paper VI)

Diaferio, A. 1999, MNRAS, 309, 610

Diaferio, A., Kauffmann, G., Balogh, M. K., et al. 2001, MNRAS, 323, 999

Dressler, A. 1980, ApJ, 236, 351

Dressler, A., \& Shectman, S. A. 1988, AJ, 95, 985

Dressler, A., Oemler, A. Jr., Sparks, W. B., et al. 1997, ApJ, 490, 577

Fasano, G., Poggianti, B. M., Couch, W. J., et al. 2000, ApJ, 452, 673

Gavazzi, G., Scodeggio, M., Boselli, A., \& Trinchieri, G. 1991, ApJ, 382,19

Gebhardt, K., Pryor, C., Williams, T. B., \& Hesser, J. E. 1994, AJ, 107, 2067

Ghigna, S., Moore, B., Governato, F., et al. 1998, MNRAS, 300, 146

Gill, S. P. D., Knebe, A., \& Gibson, B. K. 2004 [arXiv: astro-ph/0404427]

Graham, A. W., Jerjen, H., \& Guzmán, R. 2003, AJ, 126, 1787

Gunn, J. E., \& Gott, J. R. III 1972, ApJ, 176, 1

Jerjen, H., Kalnajs, A., \& Binggeli, B. 2000, A\&A, 358, 845

Katgert, P., Mazure, A., Perea, J., et al. 1996, A\&A, 310, 8 (Paper I)

Katgert, P., Mazure, A., den Hartog, R., et al. 1998, A\&AS, 129, 399 (Paper V)

Katgert, P., Biviano, A., \& Mazure, A. 2004, ApJ, 600, 657 (Paper XII)

Kent, S. M., \& Gunn, J. E. 1982, AJ, 87, 945

Lanzoni, Ciotti, L., Cappi, A., Tormen, G., \& Zamorani, G. 2004, ApJ, 600,640

Łokas, E., \& Mamon, G. A. 2001, MNRAS, 321, 155

Łokas, E., \& Mamon, G. A. 2003, MNRAS, 343, 401

Mahdavi, A., Geller, M. J., Böhringer, H., Kurtz, M. J., \& Ramella, M. 1999, ApJ, 518, 69
Mazure, A., Biviano, A., Katgert, P., Thomas, T., \& Adami, C. 2000, in Galaxy Dynamics: from the Early Universe to the Present, ed. F. Combes, G. A. Mamon, \& V. Charmandaris, ASP Conf. Ser., 197, 373

Menci, N., \& Fusco-Femiano, R. 1996, ApJ, 472, 46

Merrifield, M. R. 1998, MNRAS, 294, 347

Merritt, D. 1987, ApJ, 313, 121

Mohr, J. J., Geller, M. J., Fabricant, D. G., Wegner, G., \& Thorstensen, J. 1996, ApJ, 470, 724

Moore, B., Lake, G., Quinn, T., \& Stadel, J. 1999, MNRAS, 304, 465

Moss, C., \& Dickens, R. J. 1977, MNRAS, 178, 701

Natarajan, P., \& Kneib, J.-P. 1996, MNRAS, 283, 1031

Navarro, J. F., Frenk, C. S., \& White, S. D. M. 1997, ApJ, 490, 493

Pryor, C., \& Geller, M. J. 1984, ApJ, 278, 457

Ramella, M., Geller, M. J., \& Huchra, J. P. 1987, ApJ, 344, 57

Ramírez, A. C., \& de Souza, R. E. 1998, ApJ, 496, 693

Ramírez, A. C., de Souza, R. E., \& Schade, D. 2000, ApJ, 533, 62

Rines, K., Geller, M. J., Kurtz, M. J., \& Diaferio, A. 2003, AJ, 126, 2152

Rood, H. J., \& Turnrose, B. E. 1968, ApJ, 152, 1057

Schaeffer, R., Maurogordato, S., Cappi, A., \& Bernardeau, F. 1993 , MNRAS, 263, L21

Sodré, L. J., Capelato, H. V., Steiner, J. E., \& Mazure, A. 1989, AJ, 97, 1279

Solanes, J. M., \& Salvador-Solé 1990, A\&A, 234, 93 ( $\left.\mathrm{S}^{2}\right)$

Solanes, J. M., Manrique, A., García-Gómez, C., et al. 2001, ApJ, 548, 97

Taylor, J. E., \& Babul, A. 2004, MNRAS, 348, 811

Thomas, T. 2004, A\&A, submitted (Paper VIII)

Thomas, T., \& Katgert, P. 2004, A\&A, submitted (Paper X)

Thomas, T., Hartendorp, M., \& Katgert, P. 2004, A\&A, submitted (Paper IX)

Tormen, G., Bouchet, F., \& White, S. D. M. 1997, MNRAS, 286, 865

Tormen, G., Diaferio, A., \& Syer, D. 1998, MNRAS, 299, 728

Tully, R. B., \& Shaya, E. J. 1984, ApJ, 281, 31

van der Marel, R. 1994, MNRAS, 270, 271

van der Marel, R., Magorrian, J., Carlberg, R. G., Yee, H. K. C., \& Ellingson, E. 2000, AJ, 119, 2038 